\title{
Comparing Performance of Different Team Selection Methods Using Fantasy League Scoring System
}

\author{
Bireshwar Bhattacharjee*, Dibyojyoti Bhattacharjee
}

Department of Statistics, Assam University, Silchar, Assam, India

Received July 2, 2021; Revised August 25, 2021; Accepted September 21, 2021

Cite This Paper in the following Citation Styles

(a): [1] Bireshwar Bhattacharjee, Dibyojyoti Bhattacharjee, "Comparing Performance of Different Team Selection Methods Using Fantasy League Scoring System," International Journal of Human Movement and Sports Sciences, Vol.9, No.6, pp. 1352-1361, 2021. DOI: 10.13189/saj.2021.090631

(b): Bireshwar Bhattacharjee, Dibyojyoti Bhattacharjee, (2021). Comparing Performance of Different Team Selection Methods Using Fantasy League Scoring System. International Journal of Human Movement and Sports Sciences, 9(6), 1352-1361. DOI: 10.13189/saj.2021.090631

Copyright @2021 by authors, all rights reserved. Authors agree that this article remains permanently open access under the terms of the Creative Commons Attribution License 4.0 International License

\begin{abstract}
Several authors of late have contributed significantly towards developing mathematical models for selecting the best possible team from a host of players in different team sports, including cricket. The performance of cricketers on the different skill sets of the game, such as batting, bowling, and fielding, is considered for selecting optimum teams. Most of these mathematical models are divided into two parts. The first part tells about a method that can quantify the performance of cricketers, and the second part tells about a method that can select a squad of players from a more extensive collection of players based on the value of their performance, taking into consideration the different constraints of team selection and expertise of players. The teams formed through different methods cannot be made to play against each other, so there is no direct way to find out which method delivers the best team. Also, no competition between optimum teams obtained through different methods was ever performed in the absence of objective methodology for such comparison.This paper attempts to compare different team selection methods and identify the best mathematical model for team selection in cricket using the fantasy league scoring system.
\end{abstract}

Keywords Cricket, IPL, Fantasy Cricket, Performance Measure, Percentile Rank

\section{Introduction}

In any team sport, individuals are organized into opposing teams that compete to win. Team members act together towards a shared objective. Team members set goals, make decisions, communicate, manage, conflict and solve problems in a supportive, trusting atmosphere to accomplish their objectives. Examples of such games/sports are basketball, volleyball, rugby, water polo, handball, lacrosse, cricket, baseball, and various forms of association games such as football and hockey [1]. In any team sport, the individual players are selected based on their expertise. In the most popular team sport, football, typically a team consists of eleven players. These eleven players can be categorized based on their skill and expertise, such as goalkeeper, midfielders, defenders, strikers. The main problem is to pick a team for a given match based on the available players considering their fitness performance in the previous match, interaction with teammates, match condition, the strength of the opponent. Certain players may be out of form but have personal abilities and qualities that make them more valuable against specific teams [2]. In such a situation picking a balanced football team is a difficult task. In cricket, selecting an optimum team is quite tricky. The cricket team is a blend of players drawn from diverse expertise. Precisely, a balanced cricket team demands the services of the captain, a wicketkeeper, a few specialist batters, including openers, all-rounders and specialist bowlers (both fast bowlers and spinners).

Consequently, much subjectivity is involved in the selection of a cricket team. Depending on the strengths and weaknesses of the opponent, pitch and weather conditions, the combination of players' viz. the number of spinners, all-rounders, specialist batters and fast bowlers are decided [3]. The main difficulty that arises here is to select an optimum team that comprises a balanced mixture of players with all specializations. Every cricket team has a captain who takes most of the decisions himself in consultation with the team management, the senior players and the coach. The team management goes for a subjective selection of players to offer the best possible XI 
from an available squad of players, which became a complex decision-making problem in certain situations. Thus, different researchers proposed several mathematical models to select an optimum team. These mathematical models are based on different methods like performance measure [4], machine learning [5] [6], data envelopment analysis [7], artificial neural network [8], integer optimisation [9] [10] [11]. However, the performance of these mathematical models in a real-life situation is not monitored to date, as the researcher does not have any control over the players and just for comparing the performance of the mathematical models, the teams cannot be made to play against each other. Therefore, this paper seeks to compare the teams' performance through different mathematical models using the fantasy league point system.

Fantasy league is an internet-based gaming platform where the participants assemble virtual teams of real cricket players by combining players from two teams before they play against each other in an actual match. Some rules of the gaming platform guard the virtual teams made by the participants. The virtual team selected by a participant scores points based on the actual performance of the selected players in the accurate match played subsequently. Thus, the different virtual teams formed by the different participants compete against each other based on the performance of the cricketers in the actual match. The performance of cricketers in the actual match is converted into points compiled and totalled in the fantasy platform [12]. The participant whose selected team score the highest points wins the fantasy league corresponding to that match. The points earned by the different virtual teams created by the fantasy league participants are tabulated in the leader board. The term 'Leader board' signifies all the users to see how the team ranks amongst all other competing teams as their points are updated every minute during the actual match. There are both free and paid contests available on fantasy league platforms in which individuals participate and try to score more points than their competitors. Also, in some fantasy leagues, an individual can participate with more than one virtual team. The players whom fantasy league participants select earn points during the actual cricket match. The total points earned by the players in the virtual team determines the points scored by that team in the fantasy league. Although each of these fantasy cricket platforms has its point distribution system, here is a glance at the point system for one of the most popular fantasy cricket platforms, My Team 11. A player in the virtual team earns two points as soon as he gets selected in the playing XI of the actual cricket match. For each run scored by a batsman, he earns one point with scope for earning some bonus points by hitting a boundary, a six, thirty runs, half-century and century. Two points get deducted if the batsman is dismissed for a duck. In the case of bowlers, for each wicket taken, 25 points are awarded. Four bonus points are awarded for bowling a maiden over. Some extra points are also there for taking 3, 4, and 5 wickets haul in a match. While fielding, for each catch taken by a player, he earns 8 points; for making each run-out happen, 10 points are awarded, and for each stumping, a wicketkeeper earns 8 points. Some bonus points are also awarded for taking 3, 4 and 5 catches in a match. Maintaining a reasonable strike rate throughout the match is appreciated by some bonus points, whereas some points get deducted if the batsman's strike rate is less than 70 and is applied only to those players who have battled for at least ten balls. Further, the fantasy league participant has to choose a captain and vice-captain for their team. The points earned by the captain gets doubled; on the other hand total points earned by the vice-captain is multiplied by 1.5. In super over, none of the players will get any points for scoring runs, taking wickets.

The strategy in this paper is to form optimum teams using the different methods before an actual cricket match. Each of these teams shall then be placed in a fantasy league gaming platform. The teams' performance in terms of their ranks among other competing teams in the fantasy league platform shall be noted. The process will be repeated for several matches so that the exercise can take the benefit of replication. The final result shall show performance of the optimum teams when placed in an actual match situation. The details on the same are explained in the methodology section of the paper.

\section{Review of Literature and Research Gap}

Traditionally, player performance is measured based on the number of runs scored or the number of wickets taken [13] [14]. But in cricket, it is important to quantify the performance of the player based on the game situation such as opposition, venue, condition of the pitch, weather conditions. Thus, taking all this in account few selected research papers related to optimum cricket team selection is reviewed below.

[15] proposed a new statistic, Runs Per Match (RM) which utilizes runs scored by the cricketer in a match and the total amount of resources used, to assess the performance of batsman and bowlers in ODI. [3] formulated a measure to select an optimum team where it introduces a measure that can quantify the performance of the cricketers into a single numerical value, which is a measure of the player's cricketing efficiency. Genetic Algorithm was applied in [8] for selecting a cricket team. Optimum cricket team is selected in [9] using the method of Evolutionary Multi-Objective Optimization. [7] saw the use of Data Envelopment Analysis (DEA) model for evaluating the performance of the players and based on the DEA score the players are classified as efficient and inefficient one. In [16] the method of performance measurement was introduced for the selection of an optimum balanced team without taking the help of any integer programming. [17] displayed how the mixed integer programming method can be used to select an Australian test cricket team. A Machine Learning Algorithm was applied in [18] to create a new index named as Deep Performance Index (DPI) to classify the cricketers. Several machine learning techniques such as k-mean clustering, Naive Bayes Classifier, linear regression, support vector machine were applied in [6] to select an ODI cricket team. In [10] cricket players performance was measured based on their ability to score runs and take wickets and finally the integer optimization is used to select an optimum team.[5] developed a methodology for selecting a cricket team applying the techniques of Machine Learning Algorithm. [19] once again applied DEA to compute 
the efficiency of a player and based on this efficiency score a cricket team is formed. [11] quantifies the performance of the player taking into consideration the consistency of a cricket player. This performance measure was then taken as a basis of integer optimization to select an optimum team. Through the application of integer optimization [20] selected a cricket team for the purpose of Dream11 Cricket team selection.

On reviewing the available literature it is observed that several methods are being developed that can perform the task of team selection. Most of these methods claim to be better in performance than their preceding models. However, no research is encountered where the problem of evaluating the performance of the different optimum team selection methods is done. In the absence of any such compilation, it becomes difficult to understand which model of optimum team selection shall be used if someone is interested to pick up teams using any one of the mathematical models. Also, finding the best method of an optimum team shall be of benefit not only for the team management but also for a large section of cricket fans who are these days participating in different fantasy league platforms. The second application of the work shall increase the probability of a participant in the fantasy league to move higher in the leader board. So, the paper is out to compare and find out the best mathematical method that one may adopt in selecting an optimum team.

\section{Objective of the Study}

From the above discussion, it is clear that there are different methods available in the literature for selecting an optimum team from a host of available cricketers. The methods differ in the statistical and analytical tools used for performance measurement of cricketers and team selection. Thus, the optimum teams formed on the same set of available players are different from each other. The optimum teams might have some common players. As the optimum teams selected using different methods differ and could not play against each other, for reasons mentioned above, there is no direct way to compare the optimum teams. Accordingly, this paper is set to attain the following objective:

- To compare different optimum cricket team selection methods using fantasy league scoring system.

\section{Methodology}

\subsection{Methods Selected}

Reviewing various literature on optimum team selection methods, five optimum team selection methods are considered for comparison. While considering the optimum team selection approaches it is seen that the methods differ from each other in terms of their methodology used. This is done so that the different methodology of team selection can be brought into consideration. The five methods thus considered are based on (i) performance evaluation (ii) integer optimization (iii) integer optimization through performance measure (iv) machine learning and (v) data envelopment analysis. These methods with the name of the corresponding authors are provided in Table 1 below:

\subsection{Description of the Methods}

I. Integer programming optimization developed by Sharp, Brettenny, Gonsalves, Lourens and Stretch [10]

1. Selecting batting indicators $Y_{1 j}$ and $Y_{2 j}$

2. Next we Combine the batting indicators

$$
U_{1 j}=\left(Y_{1 j}^{\alpha}\right)\left(Y_{2 j}^{1-\alpha}\right)
$$

3. Selecting the weighting factor $\alpha$.

4. Computation of Batting Index

$$
C_{1 j}=\left[\frac{U_{1 j}}{\sum_{j=1}^{n_{1}} U_{1 j}}\right] \times n_{1}
$$

5. Selecting bowling indicators $Y_{1 j}, Y_{2 j}$ and $Y_{3 j}$

6. Next, we combine the bowling indicators

$$
U_{2 j}=Y_{3 j}{ }^{\alpha_{1}} \times Y_{4 j}{ }^{\alpha_{2}} \times Y_{5 j}{ }^{1-\alpha_{1}-\alpha_{2}}
$$

7. Selecting the weighting factors $\alpha_{1}$ and, $\alpha_{2}$

8. Estimation of

$$
V_{2 j}=\left[k-\frac{U_{2 J}}{\sum_{j=1}^{n_{2}} U_{2 j}}\right]
$$

9. Computation of $C_{2 j}^{1}=\left[k-\frac{U_{2 J}}{\sum_{j=1}^{n_{2}} U_{2 j}}\right]>0$

10. Finally the bowling index is computed using

$$
C_{2 j}^{p+1}=\left(C_{2 j}^{p}\right)^{\sigma_{1} / \sigma_{p}}
$$

11. For all-rounder index we compute

$$
C_{3 j}^{1}=\left(C_{1 j}\right)^{\beta} \cdot\left(C_{2 j}\right)^{1-\beta}
$$

12. Finally all-rounder index is computed using

$$
C_{3 j}^{p+1}=\left(C_{3 j}^{p}\right)^{\sigma_{1} / \sigma_{q}}
$$

13. Finally a balanced team is selected using Integer Optimization.

II. Data envelopment analysis developed by Gholam and Sharma [7]

1. We consider outputs for $\mathrm{n}$ player as $y_{j}=\left(y_{1 j}, y_{2 j}, \ldots, y_{n j}\right)$

2. The DEA model for evaluating the $k^{\text {th }}$ player performance is given by,

$\max \mathrm{h}$ 
Table 1. Optimum team selection methods

\begin{tabular}{|c|c|}
\hline Name of the Methods & Developed By \\
\hline Integer Programming Optimization(M-1) & Sharp et.al.(2011) \\
Data Envelopment(M-2) & Amin et.al.(2014) \\
Performance Measure(M-3) & Bhattacharjee et.al.(2016) \\
Machine Learning(M-4) & Deep et. al.(2016) \\
Integer Optimization Through Performance Measure(M-5) & Prachi et. al.(2020) \\
\hline
\end{tabular}

$$
\begin{aligned}
& \sum_{j=1}^{n} y_{r j .} \lambda_{j}-y_{r k} ; h>0, r=1,2, \ldots, s \\
& \sum_{j=1}^{n} \lambda_{j}=1 ; \lambda_{j} \geq 0, j=1,2, \ldots, n
\end{aligned}
$$

3. Solve the model for $n$ times, one model for each player to get the efficiency score of the player $h$.

4. The Efficiency of the player is determined

If the player is efficient $h=1$, .

Efficiency of the player diminishes when $h<1$.

For inefficient players $\mathrm{h} \sim 0$.

III. Performance based method developed by Saikia, Bhattacharjee, and Radhakrishnan [4]

1. Compute the values of $x_{i j k}$.

2. Get the values of $y_{i j k}$, normalizing $x_{i j k}$.

If the $k^{\text {th }}$ attribute is positively affecting the performance of player

$$
y_{i j k}=\frac{x_{i j k}-\min \left(x_{i j k}\right)}{\max \left(x_{i j k}\right)-\min \left(x_{i j k}\right)}
$$

If the $k^{\text {th }}$ attribute is negatively affecting the performance of player

$$
y_{i j k}=\frac{\max \left(x_{i j k}\right)-x_{i j k}}{\max \left(x_{i j k}\right)-\min \left(x_{i j k}\right)}
$$

3. Finding the normalizing constant

$$
\mathrm{C}_{\mathrm{k}}=\sum_{\mathrm{j}} \frac{1}{\sqrt{\operatorname{var}\left(\mathrm{y}_{\mathrm{ijk}}\right)}}
$$

4. Estimation of relative weight

$$
w_{j k}=\frac{c_{k}}{\sqrt{\operatorname{var}\left(y_{i j k}\right)}}
$$

5. Calculation of performance score for the $i^{\text {th }}$ player with respect to $k^{\text {th }}$ attribute

$$
S_{i k}=\sum_{j=1}^{n_{j}} w_{j k} y_{i j k}
$$

6. Estimation of Performance Score by

$$
S_{i}=S_{i 1}+S_{i 2}+S_{i 3}^{a_{i}}+S_{i 4}^{1-a_{i}}-1
$$

Where,

$a_{i}=1(0)$, if the $i^{\text {th }}$ player is a bowler (wicketkeeper)

7. Computation of performance measure of the $i^{\text {th }}$ player

$$
P_{i}=\frac{S_{i}}{\max \left(S_{i}\right)}
$$

\section{Machine learning based method developed by Prakash, Patvardhan and Singh [6]}

1. Selection of different attribute for batsman and bowler.

2. Calculation of

$$
p_{i j k}=\frac{\left(N-r_{i j k}\right)}{N}
$$

Where,

$r_{i j k}$ be the rank of the $i^{\text {th }}$ player in $j^{\text {th }}$ attribute (batting strike rate, bowling strike etc.) in the $k^{t h}$ discipline (i.e. batting, bowling etc.)

3. Estimation of $M V P I_{i j k}$.

$$
\begin{aligned}
& M V P I_{i j k}=\frac{(P A * R S)}{T B A}+\frac{(P S R * P S R * R S)}{(T S R * T S R)}(\text { If } k=1=\text { Bats }- \\
& \text { man }) \\
& M V P I_{i j k}=\frac{(T O A * W T)}{P A}+\frac{(T E R * T E R * W T)}{(P E R * P E R)} \text { (If } k=2= \\
& \text { Bowler) }
\end{aligned}
$$

4. Random forest algorithm used to get weight $x_{j k}$ using independent variable as MVPI.

5. Computation of relative weight

$$
w_{j k}=\frac{R I\left(x_{j k}\right)-\operatorname{Min}\left(x_{j k}\right)}{\operatorname{Max}\left(x_{j k}\right)-\operatorname{Min}\left(x_{j k}\right)}
$$

6. Estimation of player performance

$$
P P_{j k}=\sum_{j} p_{i j k .} w_{j k}
$$

7. Calculation of all-rounder performance $A R=P P_{j 1}+$ $P P_{j 2}$ 


\section{Integer optimization based on performance measure method developed by Agarwal and Talari. [11]}
1. Compute batting attributes $A V G_{k}^{B a t}$ CONSIST $T_{k}^{\text {Bat }}$..

2. Compute

$$
U_{k}^{B A T}=\left(A V G_{k}^{B a t}\right)^{\alpha} \cdot\left(C O N S I S T_{k}^{B a t}\right)^{1-\alpha}
$$

3. Next, batting index is computed

$$
B I_{K}^{B A T}=\left(\frac{U_{k}^{B A T}}{\sum_{k=1}^{n} U_{k}^{B A T}}\right) \times n .
$$

4. Selecting bowling attributes

$$
A V G_{k}^{\text {Bowl }}, C O N_{k}^{\text {Bowl }} \text { and } E C O_{k}^{\text {Bowl }}
$$

\section{Computation of}

$$
V_{k}^{B O W L}=\left(A V G_{k}^{B o w l}\right)^{\alpha_{1}} \cdot\left(C O N_{k}^{B o w l}\right)^{\alpha_{2}} \cdot\left(E C O_{k}^{B o w l}\right)^{1-}
$$

6. Finally Bowling index is computed

$$
B I_{k}^{B O W L}=\left[\frac{V_{k}^{B O W L}}{\sum_{k=1}^{n} V_{k}^{B O W L}}\right] \times m
$$

7. All-Rounder Index is computed

$$
A_{k}^{\text {All-Rounder }}=\left[U_{k}^{B A T}\right]^{\beta} \cdot\left[V_{k}^{B O W L}\right]^{1-\beta}
$$

8. Finally an optimum team is selected using Integer Programming Optimization.

Details discussions of the notations used in describing various methods are provided in Appendix Section

\subsection{Data Collection Process and Selection Criteria}

In the thirteen season of IPL played in the year 2020, 56 matches were played in the league stage of the tournament. All these matches were complete and there was no truncation of matches because of bad weather or any other reason. The first 28 league matches of IPL 2020 were considered for gauging the performance of players. Accordingly, based on the data of the players in those 28 matches the optimum teams were selected for each of the remaining 28 matches of the tournament. For example, if team X and Team Y are playing against each other on the $29^{\text {th }}$ league match of IPL 2020, then from the squad of players of Team X and Team Y optimum teams are selected by each of the five methods mentioned above. These five teams are then placed in the Fantasy League Platform "MyTeam 11" 1 As the $29^{\text {th }}$ match progresses the five

\footnotetext{
${ }^{1}$ MyTeam 11 is a fantasy cricket platform founded by Vinit Godara and Sanjit Sihag in February 2016. It is the second-largest market leader with more than 18 million users. Apart from cricket, it also started 10 other fantasy sports such as football, kabaddi, hockey etc. Many former cricketers and commentators have joined MyTeam 11 as their brand ambassadors viz. Virendra Sehwag, Danny Morrison, Mayanti Langer, Sanjana Ganeshan, Jatin Sapru etc.
}

optimum teams start earning points along with several other teams selected by other cricket fans who participated in the league based on the actual performance of cricketers on the $29^{\text {th }}$ match. At the end of the match, the rank of each of the optimum teams is noted. This process is repeated for each of the 28 matches of the second half of the league (i.e from $29^{t h}$ to $56^{\text {th }}$ match). In MyTeam 11 there are several competitions, based on the same match that one can join. The different competitions are distinguished by the fee charged (in the case of paid services) and free services. This work placed the optimum teams in a competition named "Practice Contest with 100 spots" in MyTeam 11 which is a free service where a maximum of 100 fantasy teams can participate. But an individual is not allowed to place more than five fantasy teams.

For Selecting the teams based on the methods discussed in Table 1. we collected data from cricbuzz.com for the first 28 league matches of the Indian Premier League(IPL) 2020. Since the quality of the cricket players cannot be judged from one or two games, thus the performance of the cricketers needs to be evaluated over a more significant number of matches, as the effect of extreme performances gets even out for a more significant number of games [21]. Based on their requirement, the different researchers used various benchmarks for populating players in the consideration set, i.e. the squad from which the players are selected for the optimum teams. The criteria must be selected so that along with the sufficient number of matches played, the player must demonstrate a reasonable amount of skill concerning batting, bowling and fielding. Also, among the players of the consideration set, there must be some balance between batters, bowlers (both spinners and pacers), wicket keepers and all-rounders from which the optimum teams are selected [3]. Thus, after several trials, the following criteria are decided which is provided in Table 2 to include the players in the consideration set, i.e. the squad based on the performance in the first 28 matches of IPL 2020.

The feet mentioned in Table 2 is to be attained by the players in the first 28 matches of IPL 2020. In case this is attained by a player, then he shall be considered for selection in the optimum teams of the different methods, in the matches in which his team figures.

\subsection{Virtual Cricket Team Selection in Fantasy League}

A fantasy sport is a game, often played using the Internet, where participants assemble imaginary or virtual teams composed of proxies of real players of a professional sport. Fantasy cricket is a part of the fantasy sports genre. It is an online game in which a virtual team of real cricket players is created, scoring points depending on how they perform in real-life matches. To win a tournament, players must work towards attaining the maximum points and the highest rank on the leaderboard [22]. The virtual team is formed before a real cricket match with a combination of players only from each team playing the match. Some restrictions are also there while forming the virtual team; the first restriction is that from one of the playing teams, a maximum of seven players is allowed in the virtual team, the second restriction is imposed while selecting the number of a 
Table 2. Criteria for inclusion of players in the consideration set

\begin{tabular}{|c|c|c|}
\hline Batsman & Bowlers & All-Rounders \\
\hline Scored Atleast 100 runs & Taken Atleast 5 Wickets & Scored Atleast 70 Runs \\
Played Atleast 60 Balls & Bowled Atleast 8 Overs & Played atleast 40 Balls \\
Dismissed Atleast Once & & Dismissed Atleast Once \\
& & Taken Atleast 5 Wickets \\
& & Bowled Atleast 8 Overs \\
\hline
\end{tabular}

batsman, bowlers, all-rounders and wicket-keepers that are to be included. Different fantasy league platforms have their restriction while selecting players. Thus, in this section, the binary integer optimization technique is used for selecting an optimum team. Let $s_{i}$ be the performance score for the $i^{\text {th }}$ player, and $\theta_{i}$ is the indicator variable that indicates the selection and non-selection of a particular player. Let us now define number of wicket keepers as $w_{i}$, batsmen as $b_{i}$, bowlers as $c_{i}$, and all-rounders as $a_{i}$. Thus, the objective function for the binary integer optimization to be maximized is,

Maximise

$\mathrm{G}=\sum_{i=1}^{11} \theta_{i} s_{i} \ldots(1)$ Where,

$\theta_{i}=1(0) ;$ if the $i^{t h}$ player is selected in the team(otherwise)

Subject to the constraints

$1 \leq w_{i} \leq 4$ (not less than one and not more than four

wicket-keepers in the virtual team)

$1 \leq b_{i} \leq 6$ (not less than one and not more than six batsman in the virtual team)

$1 \leq c_{i} \leq 6$ (not less than one and more than six bowlers in the virtual team)

$1 \leq a_{i} \leq 4$ (not less than one and more than four all-rounders in the virtual team)

$\sum_{i=1}^{11} p_{i} \leq 100$ (sum of the points associated with each player is less than or equal to 100 in the virtual team)

\subsection{Comparing Teams}

For evaluating the performance of the optimum team selection methods discussed above, the fantasy league scoring system of MyTeam11 [23] is used. An optimum team is constructed and placed in My Team 11's "Practice Contest with 100 spots "for each method. The teams' performance is then monitored, and the rank of each team is noted down at the end of the match. The process is repeated for each of the last 28 league matches of IPL 2020. The tool adopted to find out the best team among the selected ones is that of percentile rank[24]. The procedure adopted for the calculation of percentile rank is discussed below

- Arranged the points scored by each method in ascending order.

- Rank all the methods arranged in ascending order, denote this by $(\mathrm{M})$.

- Identify the total number of ranks, denote this by (Y).

- Identify if there is the same score as M if so count the same score as " $\mathrm{R}$ "
- If $\mathrm{R}$ not equals to 0 then,

$$
\text { Percentile Rank }(P R)=\frac{M+(0.5 * R)}{Y} \times 100 \ldots
$$

- If $\mathrm{R}$ equals to 0 then,

$$
\text { Percentile Rank }(P R)=\frac{M}{Y} \times 100 \ldots(3)
$$

- Average Percentile Rank (APR) is obtained for the 28 matches for each method using the formula,

$$
A P R=\frac{\text { Percentile Rank }}{\sum_{t=1}^{28} \text { Percentile Rank }} \ldots \text { (4) }
$$

- An Higher value of Average Percentile Rank tells us that method is a good one.

\section{Results}

Before, the match between 'Team X' and 'Team Y', the optimum virtual teams based on the five methods are selected and is placed in the fantasy league platform MyTeam XI. As the actual match progresses, the virtual teams earn points, and at the end of the match, the final earnings of the virtual teams are obtained. Table 3 below provides the fantasy score of the virtual teams selected based on different methods for match number 29 of IPL 2020 played between Sunrisers Hyderabad and Mumbai Indians.

\section{Discussion}

From Table 3, we conclude that the method that performed well is M-4, based on machine learning with a fantasy score of 478.75 and percentile rank of 100 . The exercise elaborated in Table 3 is repeated for the next 27 matches of IPL 2020. The fantasy score for each team is then noted down, and using the equation (2) or (3), percentile rank is computed for each match. Finally, equation (4) is used to compute the value of the Average Percentile Rank for each method. A higher value of the Average Percentile Rank signifies the method is a better one. Different methods with their Average Percentile Rank is elaborated in Table 4. From Table 4, it can be concluded that the Integer Programming Optimization [10] has emerged as the best method among the selected ones with an average percentile rank of 70.71. The second best method is based on Performance Measure [4] with an average percentile rank of 60. The method based on Machine Learning [6] is the thirdbest with an average percentile rank of 59.29. Figure 1 explains 
Table 3. Performance score for each methods

\begin{tabular}{||c|c|c|c|c|c||}
\hline Players Name & M-1 & M-2 & M-3 & M-4 & M-5 \\
\hline S Watson & 51 & 51 & 51 & 51 & 102 \\
A Rayudu & & & 52 & 52 & 52 \\
D Warner & & & 28.5 & 28.5 & \\
K Williamson & & & 75.5 & 75.5 & \\
MS Dhoni & 33 & 33 & 33 & 33 & 33 \\
R Jadeja & & & 72.25 & 72.25 & \\
DJ Bravo & 70 & & 70 & 70 & 70 \\
R Khan & 19 & 19 & 38 & 38 & 19 \\
T Natarajan & & & 47 & 47 & \\
P Chawla & & & 2 & 2 & 2 \\
D Chahar & 2 & 2 & 2 & & \\
S Nadeem & 14.25 & 14.25 & & 9.5 & 9.5 \\
J Bairstow & 70 & 70 & & & \\
F DuPlessis & 2 & 2 & & & 2 \\
M Pandey & 15 & 15 & & & 30 \\
V Shankar & 16 & 16 & & & \\
K Sharma & & 51 & & & 51 \\
K Ahmed & 50 & & & & \\
S Curran & & 70.75 & & & 17.5 \\
P Garg & & & & $\mathbf{3 8 8}$ \\
TOTAL & $\mathbf{3 4 2 . 2 5}$ & $\mathbf{3 4 4}$ & $\mathbf{3 7 1 . 2 5}$ & $\mathbf{4 7 8 . 7 5}$ & $\mathbf{1}$ \\
RANK & $\mathbf{5}$ & $\mathbf{4}$ & $\mathbf{2}$ & $\mathbf{1}$ & $\mathbf{3}$ \\
PR & $\mathbf{2 0}$ & $\mathbf{4 0}$ & $\mathbf{8 0}$ & $\mathbf{1 0 0}$ & $\mathbf{6 0}$ \\
\hline
\end{tabular}

Table 4. Optimum team with fantasy score

\begin{tabular}{||c|c|}
\hline Methods & Average Percentile Rank \\
\hline Integer Programming Optimization(M-1) & 70.71 \\
Data Envelopment(M-2) & 51.43 \\
Performance Measure(M-3) & 60.00 \\
Machine Learning(M-4) & 59.29 \\
Integer Optimization Through Performance Measure(M-5) & 57.14 \\
\hline
\end{tabular}

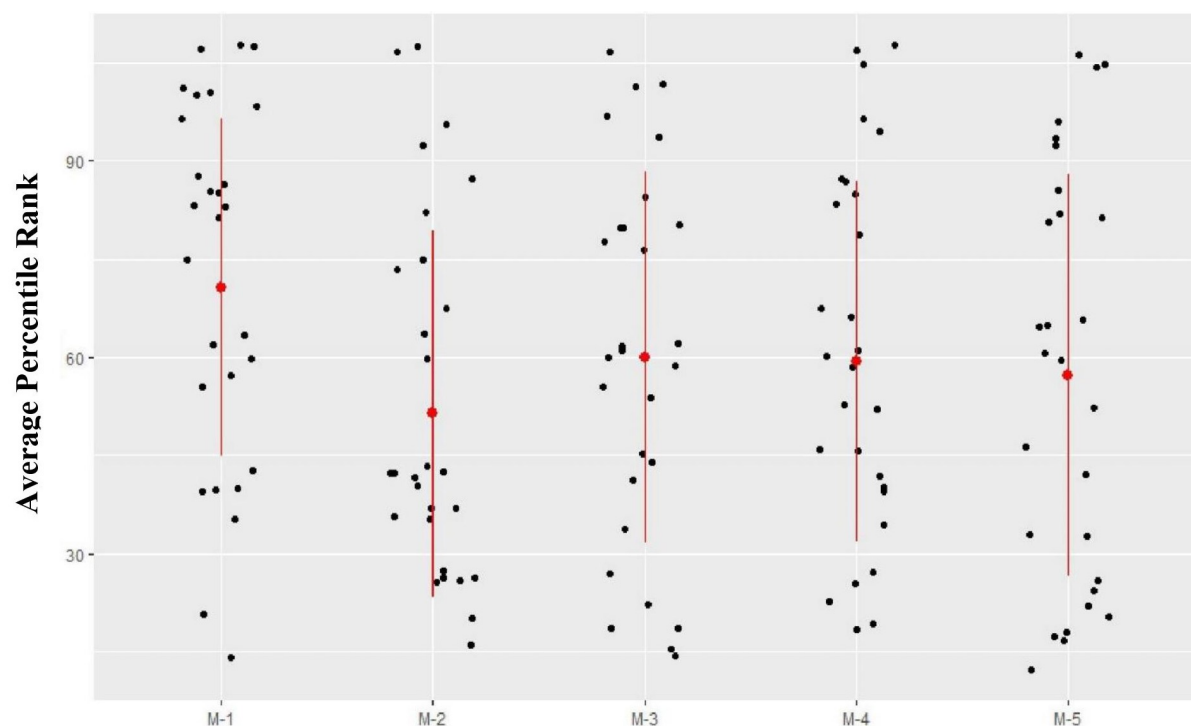

Team Selection Methods

Figure 1. Jitter plot for percentile rank of different team selection methods 
the jitter plot of percentile rank for each method discussed in Table 1] The red dot on the perpendicular line indicates the Average Percentile Rank for each method. The highest point of the red line passing through the red dot indicates the value of mean plus standard deviation, while the lowest point indicates the value of mean minus standard deviation. The figure shows that based on average percentile rank, M-1 is best, with M-3 as the second-best method. Also, for M-2 and M-5, the black dots are more scattered, which indicates that variations concerning percentile rank for these methods are more, making these methods relatively inconsistent compared to others.

\section{Conclusion}

The study enables us to compare and find the best method between the different methods selected for fantasy league team selection that one can adopt while selecting an optimum cricket team. Based on this study, we suggest that integer programming optimization is the superior one. The fantasy league participant can build his team by taking a clue out of this research work so that his team attains the leader board in the fantasy league platform. The limitation of this study is that the paper completely ignores factors like batting position, pitch condition, run-chase or target building scenarios and focuses on only one tournament. In future, the researchers can study and compare the performance of these team selection methods by addressing these issues. Similar studies can also be conducted for other sports like hockey, rugby, soccer, baseball.

\section{Conflict of Interest}

The authors declare that the research was conducted in the absence of any commercial or financial relationships that could be construed as a potential conflict of interest.

\section{REFERENCES}

[1] Wikipedia, "Team Sport." https://en.wikipedia. org/wiki/Team_sport, 2021. [Online; accessed 25March-2021].

[2] K. K. HAUGEN, "Equilibrium team selection in football under win or profit maximisation," Math. Appl, vol. 6, pp. 161-170, 2017.

[3] D. Bhattacharjee and H. Saikia, "An objective approach of balanced cricket team selection using binary integer programming method," Opsearch, vol. 53, no. 2, pp. 225247, 2016.

[4] H. Saikia, D. Bhattacharjee, and U. K. Radhakrishnan, "A new model for player selection in cricket," International Journal of Performance Analysis in Sport, vol. 16, no. 1, pp. 373-388, 2016.
[5] V. Pujbai, R. Chaudhari, D. Pal, K. Nhavi, and N. Shimpi, "A survey on team selection in game of cricket using machine learning," International Research Journal of Engineering and Technology, vol. 6, no. 11, 2019.

[6] C. D. Prakash, C. Patvardhan, and S. Singh, "A new machine learning based deep performance index for ranking ipl t20 cricketers," International Journal of Computer Applications, vol. 137, no. 10, pp. 42-49, 2016.

[7] G. R. Amin and S. K. Sharma, "Cricket team selection using data envelopment analysis," European journal of sport science, vol. 14, no. sup1, pp. S369-S376, 2014.

[8] S. Omkar and R. Verma, "Cricket team selection using genetic algorithm," in Proceedings of the International Congress on Sport Dynamics, pp. 1-9, Citeseer, 2003.

[9] F. Ahmed, K. Deb, and A. Jindal, "Multi-objective optimization and decision making approaches to cricket team selection," Applied Soft Computing, vol. 13, no. 1, pp. 402-414, 2013.

[10] G. Sharp, W. Brettenny, J. W. Gonsalves, M. Lourens, and R. Stretch, "Integer optimisation for the selection of a twenty20 cricket team," Journal of the Operational Research Society, vol. 62, no. 9, pp. 1688-1694, 2011.

[11] P. Agrawal and T. Ganesh, "Selection of indian cricket team in odi using integer optimization," in Journal of Physics: Conference Series, vol. 1478, p. 012001, IOP Publishing, 2020.

[12] Wikipedia, "Fantasy Sport." https://en.wikipedia. org/wiki/Fantasy_sport\#: : text=A $\% 20$ fantasy $\%$ 20 sport $\% 20$ (also $\% 20$ known, players $\% 20$ of $\% 20 a \%$ 20professional\%20sport./, 2021. [Online; accessed 05-January-2021].

[13] A. C. Kimber and A. R. Hansford, "A statistical analysis of batting in cricket," Journal of the Royal Statistical Society: Series A (Statistics in Society), vol. 156, no. 3, pp. 443-455, 1993.

[14] H. H. Lemmer, "A method for the comparison of the bowling performances of bowlers in a match or a series of matches," South African Journal for Research in Sport, Physical Education and Recreation, vol. 27, no. 1, pp. 75-87, 2005.

[15] T. Swartz, "The best batsmen and bowlers in one-day cricket," South African Statistical Journal, vol. 37, no. 2, pp. 203-222, 2003.

[16] D. Bhattacharjee and H. Saikia, "On performance measurement of cricketers and selecting an optimum balanced team," International Journal of Performance Analysis in Sport, vol. 14, no. 1, pp. 262-275, 2014.

[17] H. Thorburn, "Team Selection." https://vrs.amsi.org.au/ decision-making-and-cricket-team-selection/, 2015. [Online; accessed 05-January-2021]. 
[18] M.-B. Malek, R. H. Badhan, M. I. Shesir, and N. H. Fakir, Squad selection for cricket team using machine learning algorithms. PhD thesis, Brac University, 2018.

[19] R. Chaudhary, S. Bhardwaj, and S. Lakra, "A dea model for selection of indian cricket team players," in 2019 Amity International Conference on Artificial Intelligence (AICAI), pp. 224-227, IEEE, 2019.

[20] S. Singla and S. S. Shukla, "Integer optimisation for dream 11 cricket team selection," 2020.

[21] A. Lewis, "Towards fairer measures of player performance in one-day cricket," Journal of the Operational Research Society, vol. 56, no. 7, pp. 804-815, 2005.

[22] Wikipedia, "Fantasy Sport." https://en.wikipedia. org/wiki/Fantasy_cricket/
[23] MyTeam11, "Fantasy Sport Scoring System." https: //www .myteam11.com/fantasy-points-system . html\# : : text=MyTeam 11\%20allows $\% 20 a \% 20$ total $\%$ 20of , the \%20play\%20on\%20the\%20field./.

[24] L. Thurstone, "The calculation and interpretation of percentile ranks," The Journal of Educational Research, vol. 6, no. 3, pp. 225-235, 1922.

\section{Appendix}


Table 5. Notations Related to Method No. 1(M-1)

\begin{tabular}{|c|c|}
\hline Notation & Description \\
\hline$x_{i j k}$ & Performance Score of $i^{\text {th }}$ player in the $j^{\text {th }}$ discipline for the $k^{\text {th }}$ attribute \\
\hline$y_{i j k}$ & Normalized value of batting, bowling fielding factors etc. \\
\hline$w_{j k}$ & Weights for different factors viz. batting, bowling etc. \\
\hline$S_{i 1}$ & Performance score for Batting \\
\hline$S_{i 2}$ & Performance score for Fielding \\
\hline$S_{i 3}$ & Performance score for Bowling \\
\hline$S_{i 4}$ & Performance Score for Wicket Keeper \\
\hline$a_{i}$ & $=1, i f i^{\text {th }}$ player is a Bowler \\
\hline & $=0$, if $i^{\text {th }}$ player - is - - - Wicket - Keeper \\
\hline
\end{tabular}

Table 6. Notations Related to Method No. 2 (M-2)

\begin{tabular}{|c|c|}
\hline Notation & Description \\
\hline$P A$ & Player Average \\
\hline$R S$ & Runs Scored \\
\hline TBA & Tournament Batting Average \\
\hline PSR & Player Strike Rate \\
\hline$T S R$ & Tournament Strike Rate \\
\hline$T O A$ & Tournament Bowling Average \\
\hline$W T$ & Wickets Taken \\
\hline$P A$ & Player Average \\
\hline$T E R$ & Tournament Economy Rate \\
\hline$P E R$ & Player Economy Rate \\
\hline$r_{i j k}$ & Rank of $i^{\text {th }}$ player in ${ }^{\text {th }}$ attribute in the $k^{\text {th }}$ discipline \\
\hline$M V P I_{i j k}$ & Most Valuable Player Index for the $i^{\text {th }}$ player in $j^{\text {th }}$ attribute in the $k^{\text {th }}$ discipline \\
\hline$R I$ & Relative Importance \\
\hline \hline
\end{tabular}

Table 7. Notations Related to Method No. 4 (M-4)

\begin{tabular}{|c|c|}
\hline Notation & Description \\
\hline$Y_{1 j}$ & Total Number of Runs Scored by Player $\mathrm{j} /$ Total Number of Dismissal for Player $\mathrm{j}$ \\
\hline$Y_{2 j}$ & Total Number of Runs Scored by Player $\mathrm{j} /$ Total Number of Balls Received by player $\mathrm{j}$ \\
\hline$U_{1 j}$ & Combination of Batting Indicators \\
\hline$C_{1 j}$ & Batting Index \\
\hline$Y_{3 j}$ & Total Number of Runs Conceded by Bowler $\mathrm{j} /$ Total Number of Overs Bowled by Bowler $\mathrm{j}$ \\
\hline$Y_{4 j}$ & Total Number of Runs Conceded by Bowler $\mathrm{j}$ / Total Number of Wickets Taken by Bowler $\mathrm{j}$ \\
\hline$Y_{5 j}$ & Total Number of Balls Bowled by Bowler $\mathrm{j} /$ Total Number of Wickets Taken by Bowler $\mathrm{j}$ \\
\hline$U_{2 j}$ & Combination of Bowling Indicators \\
\hline$V_{2 j}$ & Bowling Index \\
\hline$C_{2 j}^{p+1}$ & Adjusted Bowling Index \\
\hline$\sigma_{1}$ & Standard Deviation of Batting Index \\
\hline$\sigma_{p}$ & Standard Deviation of the Bowling Index \\
\hline$C_{2 j}^{p}$ & Stand Deviation of the Bowling Index for the $p^{\text {th }}$ iteration \\
\hline$C_{3 j}^{1}$ & All-rounder index \\
\hline$C_{3 j}^{p+1}$ & Adjusted all -rounder index \\
\hline$x_{1 j}$ & Number of Batsman \\
\hline$x_{2 j}$ & Number of Bowler \\
\hline$x_{3 j}$ & Number of All-Rounder \\
\hline$x_{4 j}$ & Number of Wicket Keeper \\
\hline$c_{i j}$ & Cost Associated with that player \\
\hline &
\end{tabular}

Table 8. Notations Related to Method No. 5 (M-5)

\begin{tabular}{|c|c|}
\hline Notation & Description \\
\hline$A V G_{k}^{B a t}$ & Total Number of Runs / Total Number of Dismissals for player k \\
\hline$C O N S I S T_{k}^{B a t}$ & Standard Deviation of Player k / Batting Average of Player k \\
\hline$U_{k}^{B A T}$ & Combination of Batting Indicators for kth batsman \\
\hline$B I_{K}^{B A T}$ & Batting Index for $\mathrm{k}^{\text {th }}$ player \\
\hline$A V G_{k}^{B o w l}$ & Number of Runs Conceded by Bowler k / Number of Wickets Taken by Bowler k \\
\hline$C O N_{k}^{B o w l}$ & Standard Deviation of Bowler k/Bowling Average of Bowler k \\
\hline$E C O_{k}^{\text {Bowl }}$ & Total Number of Runs Conceded By Bowler k/Total Number of Bowlers Bowled by Bowler k \\
\hline$V_{k}^{B O W L}$ & Combination of Bowling Indicators for $k^{\text {th }}$ Bowler \\
\hline$B I_{K}^{B O W L}$ & Bowling Index for $k^{\text {th }}$ bowler \\
\hline$A_{k}^{\text {All-Rounder }}$ & All-Rounder Index for $k^{\text {th }}$ All-Rounder \\
\hline
\end{tabular}

